\title{
Monte Carlo Method to Uncertainty Evaluation of The Relative Dielectric Constant and Loss Tangent Measured by Split-Cavity Resonator Technique
}

\author{
Fei Zhao ${ }^{1, *}$, Jing $\mathrm{Pei}^{1}$, Qi Tang ${ }^{2}$, Chengxiang Wang ${ }^{3}$ and Jinsong Kan ${ }^{1}$ \\ ${ }^{1}$ Metrology and Testing Center, China Electronics Standardization Institute, Beijing 100176, China \\ ${ }^{2}$ School of Electronic Engineering, Xi' an University of Posts \& Telecommunications, Xi' an 710121, China \\ ${ }^{3}$ Shandong Qinghua Tongfang Luying Electronic Co., Ltd., Yinan County Town 276300, Shandong, China
}

\begin{abstract}
According to the mathematical model of the split-cylinder resonator method, it is impossible to establish a simple equation between the intermediate variables and the relative dielectric constant or the loss tangent. Instead, the measured relative dielectric constant and the loss tangent must be calculated numerically by iterative programming. When evaluating uncertainty according to the Guide to the Expression of Uncertainty in Measurement (GUM), it is impossible to obtain the sensitivity coefficient of each intermediate. Therefore, the reliability of uncertainty evaluation is greatly reduced. ISO/TS15530 states that the most effective method for estimating uncertainty is computer simulation, and more specifically, a Monte Carlo simulation. In this study, Three typical samples with different relative dielectric constant and loss tangent were measured, respectively. The probability density function of each intermediate variable was given, according to which the Monte Carlo simulations were performed by MATLAB programming. Moreover, some of the simulation results are compared with those of the previous literature, and the results show that the measurement uncertainty evaluated by the GUM method was slightly larger, indicating that it was a little bit conservative to assume that each intermediate variable is completely uncorrelated in the GUM method.
\end{abstract}

\section{Introduction}

For measuring the relative dielectric constant and loss tangent of low-loss dielectric substrates nondestructively, The split-cylinder resonator technique method was originally proposed by G. Kent [1]. Janezic and Baker-Jarvis [2] made a full-wave analysis of the split cylinder resonator and outlined a model where the fringing fields are rigorously accounted for and the resonance condition is derived. Moreover, by introducing a proper boundary, they enclosed the entire split-cylinder resonator fixture, thereby enabling them to derive a valid resonance condition using the modematching method without introducing a systematic error in the calculation of the relative dielectric constant and loss tangent of the samples [3,4]. Fang and Linton et al. $[5,6]$ also suggested a developed apparatus and made a systematic analysis based on a rigorous mode match model.

According to the Guide to the Expression of Uncertainty in Measurement (GUM), the uncertainty of measurements was evaluated by Janezic [3], and similar uncertainty results were also obtained on the basis of our own split-cylinder resonator [7]. However, there are obvious deficiencies in the mathematical model of measurement. Both the relative dielectric constant $\left(\varepsilon_{\mathrm{r}}\right)$ and loss tangent $(\tan \delta)$ of the sample are assumed to be abstract functions of several intermediate variables. Therefore, the sensitivity coefficients of all intermediate variables cannot be directly calculated. They are all assumed to be non-related instead.

ISO/TS15530 states that the most effective method for estimating uncertainty is computer simulation, and more specifically, a Monte Carlo simulation. To the best of the knowledge of the authors, for measurement or calibration of the $\varepsilon_{\mathrm{r}}$ and $\tan \delta$ of the samples measured by a split-cylinder resonator method, there are few reports on studies that have evaluated the measurement uncertainties by Monte Carlo method (MCM) and then verified the equivalence of the measurement uncertainties by GUM and MCM. Therefore, In this study, detailed uncertainty evaluation procedure has been given by applying Monte Carlo simulation for evaluating the uncertainties of the $\varepsilon_{\mathrm{r}}$ and $\tan \delta$ of the samples measured by a split-cylinder resonator method. The Monte Carlo simulation results of three typical samples with different the $\varepsilon_{\mathrm{r}}$ and $\tan \delta$ are presented. Some of the results were compared with those of the previous literature.

\footnotetext{
* Corresponding author: cesizf@163.com
} 


\section{Methodology}

\subsection{Monte Carlo method of estimating measurement uncertainty}

The Monte Carlo method (MCM) is a tool that uses the computational capacity of current computers to simulate a high account of pseudo random numbers. In this way, it allows to simulate complex system from a probabilistic point of view. The MCM uses a large number of samples, with different probability density functions, to obtain the final uncertainty distribution. Figure 1 illustrates the propagation of distributions. The model input quantities are $X=\left(X_{1}, X_{2}, \ldots, X_{\mathrm{N}}\right)^{\mathrm{T}}$. The probability density functions (PDF) $g_{X i}\left(\xi_{i}\right)$ may be different from each other. The PDF $\mathrm{G}_{Y}(\eta)$ for the value of the output quantity $Y$ is indicated as being asymmetric, as can arise for nonlinear models. Therefore, a large set of model values obtained can be applied to provide an approximation to the distribution of possible values for the output quantity.

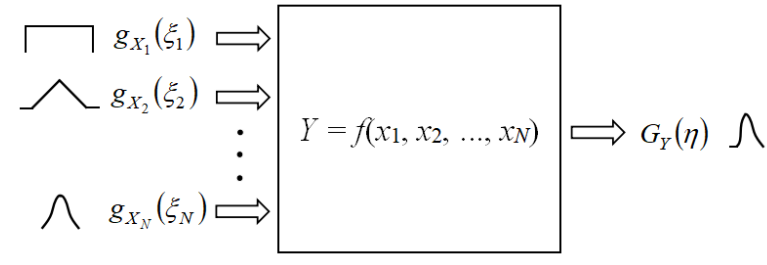

Fig. 1. Illustration of the propagation of distribution based on MCM.

An implementation procedure of the propagation of distributions based on MCM can be stated as a step-bystep procedure:

1) The model $Y=f\left(X_{i}\right)$ shall be established at the formulation stage of uncertainty evaluation;

2) The probability density functions for the input quantities $X_{i}, g_{X i}\left(\xi_{i}\right)$ shall be assigned;

3) The number of $M$ of Monte Carlo trails to be made shall be selected;

4) The required coverage probability $p$ (e.g. $95 \%$, or 0.95 ) shall be determined;

5) $M$ samples of the (set of $N$ ) input quantities shall be generated;

6) A model giving the corresponding output quantity value shall be evaluated for each sample;

7) The values of the output quantity shall be sorted into non-decreasing order, using the sorted values to approximate the distribution function for the output quantity value;

8) The estimate of the output quantity value and the associated standard uncertainty from the distribution function shall be formed;

9) The shorted $p$ coverage interval for the output quantity value from this distribution function shall be formed.

For more details about the MCM of estimating measurement uncertainty, one can refer to Ref [8-10] or other related literature.

\subsection{Split-cylinder Resonator techniques}

The principal drawing of the split-cylinder resonator is shown in Figure 2. A planar dielectric sample is placed in the variable-length gap between the two shorted cylindrical waveguide sections. In order to excite the $\mathrm{TE}_{0 \mathrm{np}}$ family of resonant modes, coupling loops in both waveguide sections are connected to the input ports of a network analyzer via coaxial transmission lines. From measurements of the resonant frequency and quality factor of the $\mathrm{TE}_{0 \mathrm{np}}$ resonant modes, the $\varepsilon_{\mathrm{r}}$ and $\tan \delta$ of the sample can be determined. In the model for calculating the compex dielectric constant, Janezic et al. [2-4] assumed that a perfect conductor exists at some sufficiently large $\rho=b$. By introducing this boundary, they enclose the entire split-cylinder resonator fixture, thereby enabling them to derive a valid resonance condition using the mode-matching method without introducing a systematic error in the calculation of the complex dielectric constant of the sample. The derivation of the $\varepsilon_{\mathrm{r}}$ and $\tan \delta$ was given in literature [2-4]. In this work, the numerical calculation of the above derivation is realized by MATLAB programming [11, 12 ], by which the uncertainty evaluation based on MCM is also achieved.

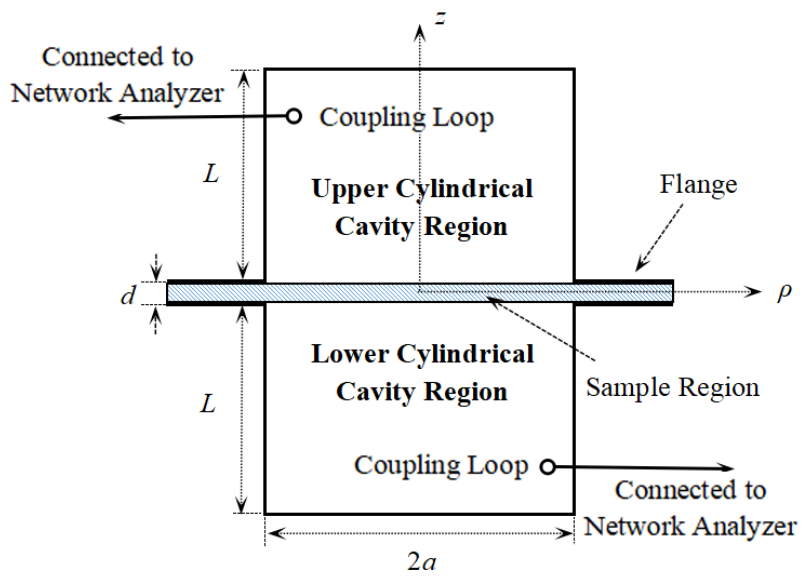

Fig. 2. Cross-sectional diagram of a split-cylinder resonator.

Three typical samples with nominal relative dielectric constant ranging from 3.8 to 77 and loss tangent ranging from $8 \times 10^{-5}$ to $4 \times 10^{-4}$ have been measured using our own split-cylinder resonator fixture, of which the nominal resonant frequency of $\mathrm{TE}_{011}$ mode is $10 \mathrm{GHz}$. An E8363B network analyzer (Agilent Technologies, CA) was used in the measurement.

\subsection{Mathematical model of the measurement}

According to the mathematical model of the splitcylinder resonator technique, the $\varepsilon_{\mathrm{r}}$ and $\tan \delta$ should be programmed for iterative numerical calculations. Therefore it is impossible to establish a simple equation between the intermediate variables and the $\varepsilon_{\mathrm{r}}$ and $\tan \delta$. However, these two parameters can be assumed as a function $f$ of several intermediate variables:

$$
\begin{gathered}
\varepsilon_{r}=f\left(f_{1}, a, L, d\right) \\
\tan \delta=f\left(f_{1}, Q_{1}, Q_{0}, a, L, d\right)
\end{gathered}
$$


where:

$f_{1}$ - the resonant frequency of the $\mathrm{TE}_{011}$ mode of an cavity with a sample

$Q_{1}$ - the quality factor of the $\mathrm{TE}_{011}$ mode of an cavity with a sample

$Q_{0}$ - the quality factor of the $\mathrm{TE}_{011}$ mode of an cavity without a sample

$a-$ cylindrical cavity radius

$L-$ cylindrical cavity length

$d$ - sample thickness

In advance of dielectric constant measurements, the cavity parameters such as cylindrical cavity radius and length are determined from the measurements for the $\mathrm{TE}_{011}$ and $\mathrm{TE}_{012}$ resonance modes of an empty cavity without a sample. The cylindrical cavity radius and length are determined from two measured resonant frequencies, $f_{01}$ for the $\mathrm{TE}_{011}$ mode and $f_{02}$ for the $\mathrm{TE}_{012}$ mode, by using the following equations [13]:

$$
\begin{gathered}
a=\frac{c j^{\prime}}{2 \pi} \sqrt{\frac{3}{4 f_{01}^{2}-f_{02}^{2}}} \\
L=\frac{c}{4} \sqrt{\frac{3}{f_{02}^{2}-f_{01}^{2}}}
\end{gathered}
$$

where:

$c$ - the velocity of light in a vacuum, $c=$ $2.9979 \times 10^{8} \mathrm{~m} / \mathrm{s}$

$j^{\prime}{ }^{01}$ - the first zero of the Bessel function of the first kind $J_{1}, j^{\prime}{ }_{01}=3.83173$

$f_{01}$ - the resonant frequency of the $\mathrm{TE}_{011}$ mode

$f_{02}$ - the resonant frequency of the $\mathrm{TE}_{012}$ mode

\section{Results and Discussion}

\subsection{The input quantity and its PDF}

Intermediate variables related to the cavity parameters are used in the $\varepsilon_{\mathrm{r}}$ and $\tan \delta$ calculation. Table 1 lists each intermediate variable and its assigned Probability density functions (PDF).

Table 1. Input values and PDF for the cavity parameters.

\begin{tabular}{|c|c|c|}
\hline $\begin{array}{c}\text { Quantity } \\
X_{i}\end{array}$ & $\begin{array}{c}\text { Probability density } \\
\text { functions }\end{array}$ & Silica \\
\hline$f_{01}$ & Rectangular & $\mathrm{R}(10017.64 \mathrm{MHz}$, \\
& distribution, $\mathrm{R}(a, b)$ & $10017.70 \mathrm{MHz})$ \\
\hline$f_{02}$ & Rectangular & $\mathrm{R}(11234.74 \mathrm{MHz}$, \\
& distribution, $\mathrm{R}(a, b)$ & $11234.80 \mathrm{MHz})$ \\
\hline
\end{tabular}

Three typical samples with different nominal relative dielectric constant and loss tangent have been measured and the intermediate variables, together with assigned PDF are listed in Table 2, Table 3 and Table 4, respectively.
Table 2. Input values and PDF for the sample Silica evaluation.

\begin{tabular}{|c|c|c|}
\hline $\begin{array}{c}\text { Quantity } \\
X_{i}\end{array}$ & $\begin{array}{c}\text { Probability density } \\
\text { functions }\end{array}$ & Silica \\
\hline$f_{1}$ & $\begin{array}{c}\text { Rectangular } \\
\text { distribution, } \mathrm{R}(a, b)\end{array}$ & $\begin{array}{c}\mathrm{R}(9339.77 \mathrm{MHz}, \\
9339.83 \mathrm{MHz})\end{array}$ \\
\hline$d$ & $\begin{array}{c}\text { Normal distribution, } \\
\mathrm{N}(\mu, \sigma)\end{array}$ & $\mathrm{N}(1.007 \mathrm{~mm}, 0.004 \mathrm{~mm})$ \\
\hline$Q_{1}$ & $\begin{array}{c}\text { Rectangular } \\
\text { distribution, } \mathrm{R}(a, b)\end{array}$ & $\mathrm{R}(12368,13133)$ \\
\hline$Q_{0}$ & $\begin{array}{c}\text { Rectangular } \\
\text { distribution, } \mathrm{R}(a, b)\end{array}$ & $\mathrm{R}(25608,27192)$ \\
\hline
\end{tabular}

Table 3. Input values and PDF for the sample K20 evaluation.

\begin{tabular}{|c|c|c|}
\hline $\begin{array}{c}\text { Quantity } \\
X_{i}\end{array}$ & $\begin{array}{c}\text { Probability density } \\
\text { functions }\end{array}$ & $\mathrm{K} 20$ \\
\hline$f_{1}$ & $\begin{array}{c}\text { Rectangular } \\
\text { distribution, } \mathrm{R}(a, b)\end{array}$ & $\begin{array}{c}\mathrm{R}(6301.56 \mathrm{MHz}, \\
6301.62 \mathrm{MHz})\end{array}$ \\
\hline$d$ & $\begin{array}{c}\text { Normal distribution, } \\
\mathrm{N}(\mu, \sigma)\end{array}$ & $\mathrm{N}(0.976 \mathrm{~mm}, 0.004 \mathrm{~mm})$ \\
\hline$Q_{1}$ & $\begin{array}{c}\text { Rectangular } \\
\text { distribution, } \mathrm{R}(a, b)\end{array}$ & $\mathrm{R}(9021,9579)$ \\
\hline$Q_{0}$ & $\begin{array}{c}\text { Rectangular } \\
\text { distribution, } \mathrm{R}(a, b)\end{array}$ & $\mathrm{R}(25608,27192)$ \\
\hline
\end{tabular}

Table 4. Input values and PDF for the sample K77 evaluation.

\begin{tabular}{|c|c|c|}
\hline $\begin{array}{c}\text { Quantity } \\
X_{i}\end{array}$ & $\begin{array}{c}\text { Probability density } \\
\text { functions }\end{array}$ & $\mathrm{K} 77$ \\
\hline$f_{1}$ & $\begin{array}{c}\text { Rectangular } \\
\text { distribution, } \mathrm{R}(a, b)\end{array}$ & $\begin{array}{c}\mathrm{R}(3445.53 \mathrm{MHz}, \\
3445.59 \mathrm{MHz})\end{array}$ \\
\hline$d$ & $\begin{array}{c}\text { Normal distribution, } \\
\mathrm{N}(\mu, \sigma)\end{array}$ & $\mathrm{N}(1.003 \mathrm{~mm}, 0.004 \mathrm{~mm})$ \\
\hline$Q_{1}$ & $\begin{array}{c}\text { Rectangular } \\
\text { distribution, } \mathrm{R}(a, b)\end{array}$ & $\mathrm{R}(2134,2266)$ \\
\hline$Q_{0}$ & $\begin{array}{c}\text { Rectangular } \\
\text { distribution, } \mathrm{R}(a, b)\end{array}$ & $\mathrm{R}(25608,27192)$ \\
\hline
\end{tabular}

\subsection{The number of Monte Carlo trials}

The number of Monte Carlo trials was set to 1000000 . Since the $\varepsilon_{\mathrm{r}}$ and $\tan \delta$ obtained by the split-cylinder resonator method are solved by numerical iterative method, it takes about 3 to 5 seconds to calculate the results of each time on a workstation (Lenovo D20; CPU: Intel Xeon E5649 $2.53 \mathrm{GHz}, 2$ processors with $12 \mathrm{~GB}$ RAM). Therefore, it would have taken about 1000 $1400 \mathrm{hrs}$ to finish totally 1000000 times simulations. In order to shorten the simulation time, the trails on the workstation were divided into 10 independent parallel calculations. As a result, the number of each trials dropped to 100000 . Even so, it still takes about 200 $220 \mathrm{hrs}$ to complete all the calculations for each samples.

\subsection{Uncertainty of relative dielectric constant}

Figure 3 shows the Monte Carlo simulation's resulting histogram with corresponding normal fit and the shortest $95 \%$ coverage interval for the $\varepsilon_{\mathrm{r}}$ of the sample Silica, $\mathrm{K} 20$ and K77, respectively. These results reveal that the 
probability density function of calibrated $\varepsilon_{\mathrm{r}}$ of all samples with different $\varepsilon_{\mathrm{r}}$ shows a normal distribution.

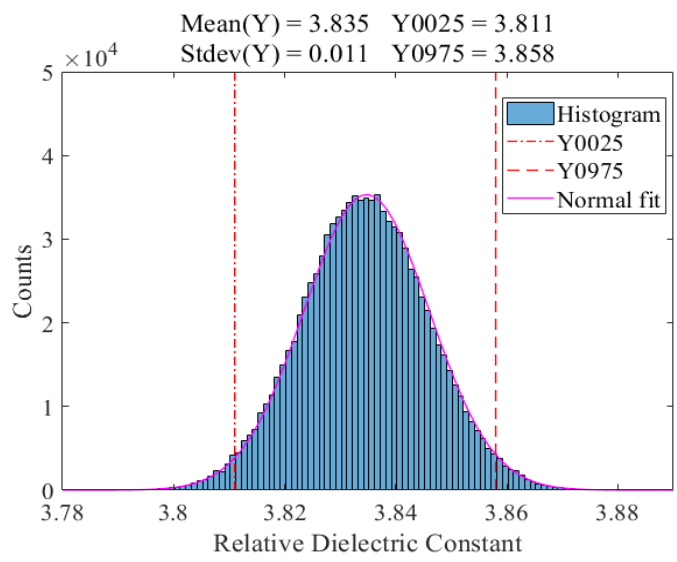

(a) Silica

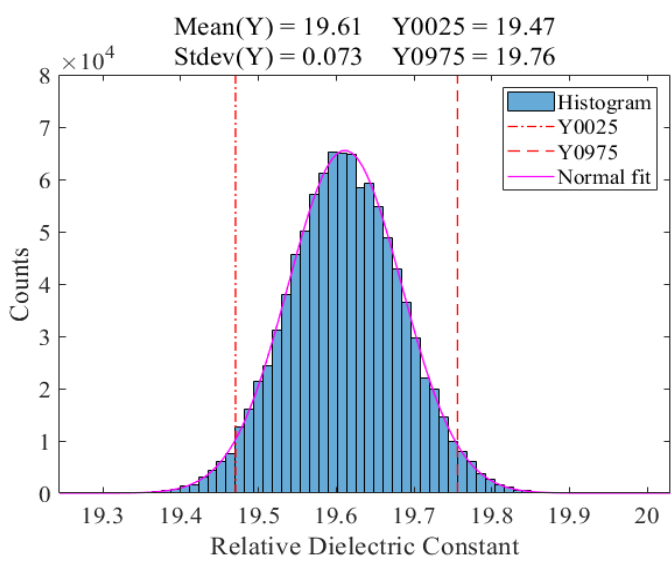

(b) K20

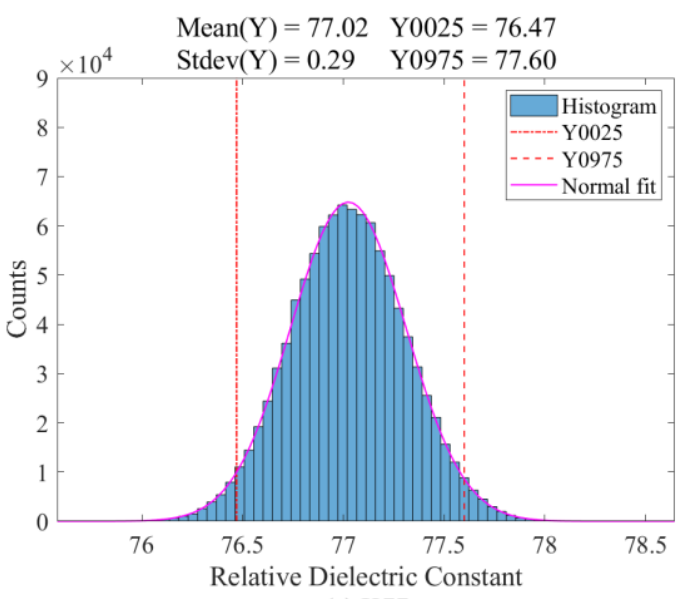

(c) K77

Fig. 3. Probability density function for relative dielectric constant calibration of the sample Silica, K20 and K77.

The calibrated relative dielectric constant for the sample Silica is 3.835 and the corresponding uncertainty is 0.024 , that is, the relative uncertainty is $0.63 \%(k=2)$. The uncertainty is much smaller than that of the GUM method[3,7]. The calibrated value for the sample K20 is 19.61 and the corresponding uncertainty is 0.15 , that is, the relative uncertainty is $0.77 \%(k=2)$. The uncertainty is also smaller than that of the GUM method obtained in our previous study [7]. These results may indicate that it was slightly conservative to assume the sensitivity coefficient of each intermediate variable is completely uncorrelated in both previous GUM method [3,7]. The calibrated value for the sample K77 is 77.02 and the corresponding uncertainty is 0.57 , that is, the relative uncertainty is $0.74 \%(k=2)$.

\subsection{Uncertainty of loss tangent}

Figure 4 illustrates the Monte Carlo simulation's resulting histogram and the shortest 95\% coverage interval for the $\tan \delta$ of the sample Silica, K20 and K77, respectively.

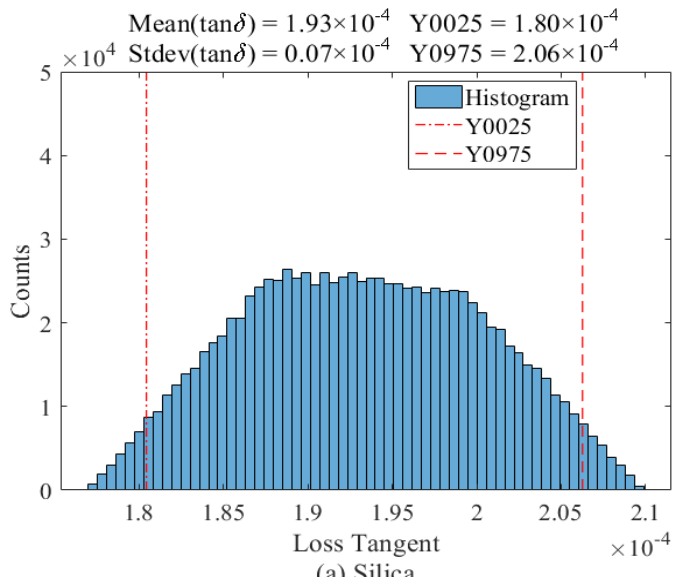

(a) Silica

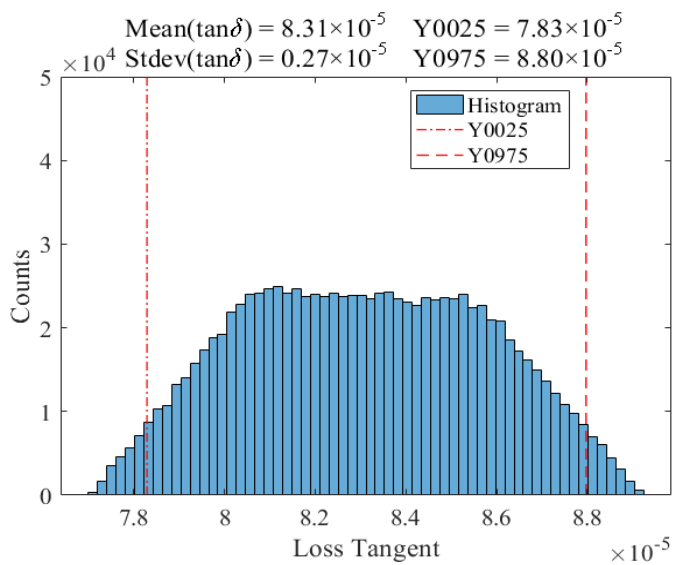

(b) $\mathrm{K} 20$

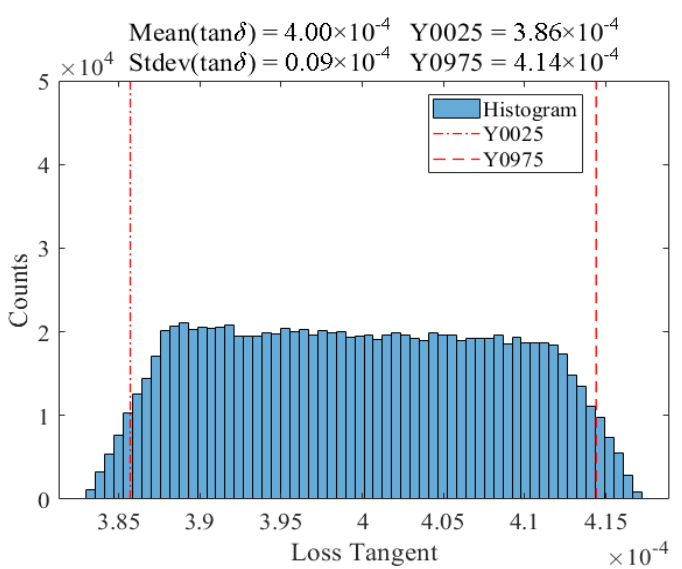

(c) K77

Fig. 4. Probability density function for loss tangent calibration of the sample Silica, K20 and K77. 
These results reveal that the probability density function of calibrated loss tangent of all samples with different loss tangent shows a trapezoidal distribution. The calibrated loss tangent for the sample Silica is $1.93 \times 10^{-4}$ and the corresponding uncertainty is $0.13 \times 10^{-4}$ $(k=2)$. The uncertainty is on the same order of magnitude as that evaluated by GUM given in Ref [3]. The calibrated loss tangent for the sample K20 is $8.3 \times 10^{-}$ 5 and the corresponding uncertainty is $0.49 \times 10^{-5}(k=2)$. The calibrated loss tangent for the sample K77 is $4.00 \times 10^{-4}$ and the corresponding uncertainty is $0.14 \times 10^{-4}$ $(k=2)$.

\section{Conclusions}

Measurement uncertainty is used to rationally characterize the dispersion of a measurement value and parameters related to measurement results, helping ensure the accuracy and reliability of measurement results and the accuracy of judgment regarding whether products conform to requirements, assisting in the acquisition of laboratory accreditation. In response to actual requirements, this paper demonstrated estimations of the uncertainty of the $\varepsilon_{\mathrm{r}}$ and $\tan \delta$ measurements by split-cylinder resonator method using Monte Carlo simulations run by MATLAB programming. In addition, some of the simulation results were compared with those of the previous literature, which showed that the measurement uncertainty evaluated by GUM method may overestimate some uncertainty components, resulting in slightly larger evaluation results.

Since the current Monte Carlo simulation process is too time-consuming, the adaptive Monte Carlo method should be further studied. If we can prove that these two methods are equivalent, from the time-consuming point of view, the adaptive Monte Carlo method may be more practical for the uncertainty evaluation method of the $\varepsilon_{\mathrm{r}}$ and $\tan \delta$ of dielectric samples.

\section{References}

1. G. Kent. An evanescent-mode tester for ceramic dielectric substrates. IEEE T. Microw. Tech. 36, 1451 (1988)

2. M.D. Janezic, J. Baker-Jarvis. Full-wave analysis of a split-cylinder resonator for nondestructive permittivity measurements. IEEE T. Microw. Tech. 47, 2014 (1999)

3. M.D. Janezic. Nondestructive relative dielectric constant and loss tangent measurement using a split-cylinder resonator $(\mathrm{Ph} . \mathrm{D}$. Thesis, University of Colorado at Boulder, 2003)

4. M.D. Janezic, E.F. Kuester, J. Baker-Jarvis. Broadband complex permittivity measurements of dielectric substrates using a split-cylinder resonator.
IEEE MTT-S Int. Microw. Symposium Digest, 1817 (2004)

5. X.Y. Fang, D. Linton, C. Walker, B. Collins. Nondestructive characterization for dielectric loss of low permittivity substrate materials. Meas. Sci. Technol. 15, 747 (2004)

6. X.Y. Fang, D. Linton, C. Walker, B. Collins. A tunable split resonator method for nondestructive permittivity characterization. IEEE T. Instrum. Meas. 53, 1473 (2004)

7. F. Zhao, P Xu, C.T. Sha, W.F. Wang, J.S. Kan. A nondestructive method for measurements of complex permittivity of microwave dielectric substrate materials. Proc. 3rd Int. Conf. Mater. Mech. Manuf. Eng. 968 (2015)

8. JCGM 104:2009. Evaluation of measurement data - An introduction to the "Guide to the expression of uncertainty in measurement" and related documents. (Joint Committee for Guides in Metrology, Sèvres, 2009)

9. JCGM 101:2008. Evaluation of measurement data - Supplement 1 to the "Guide to the expression of uncertainty in measurement" - Propagation of distributions using a Monte Carlo method. (Joint Committee for Guides in Metrology, Sèvres, 2008)

10. JCGM 102:2011. Evaluation of measurement data - Supplement 2 to the "Guide to the expression of uncertainty in measurement" - Extension to any number of output quantities. (Joint Committee for Guides in Metrology, Sèvres, 2011)

11. C.P. Robert, G. Casella, Monte Carlo Statistical Methods (Springer-Verlag, New York, 1999)

12. M. Fernández, J.M. Calderón, P.M. Bravo Díez. Implementation in MATLAB of the adaptive Monte Carlo method for the evaluation of measurement uncertainties. Accred. Qual. Assur. 14, 95 (2009)

13. IEC 62562:2010. Cavity resonator method to measure the complex permittivity of low-loss dielectric plates. (European Committee for Electrotechnical Standardization, Brussels, 2011) 